\title{
A candidate former companion star to the Magnetar CXOU J164710.2-455216 in the massive Galactic cluster Westerlund 1
}

\author{
P.J. Kavanagh ${ }^{\mathbf{1}}$ \\ School of Physical Sciences and NCPST, Dublin City University \\ Glasnevin, Dublin 9, Ireland \\ E-mail: patrick.kavanagh8@mail.dcu.ie

\section{E.J.A. Meurs} \\ School of Cosmic Physics, DIAS, and School of Physical Sciences, DCU \\ Glasnevin, Dublin 9, Ireland \\ E-mail: ejameurs@gmail.com

\section{Norci} \\ School of Physical Sciences and NCPST, Dublin City University \\ Glasnevin, Dublin 9, Ireland \\ E-mail: laura.norciegmail.com
}

Besides carrying the distinction of being the most massive young star cluster in our Galaxy, Westerlund 1 contains the notable Magnetar CXOU J164710.2-455216. While this is the only collapsed stellar remnant known for this cluster, a further $\sim 10^{2}$ Supernovae may have occurred on the basis of the cluster Initial Mass Function, possibly all leaving Black Holes. We identify a candidate former companion to the Magnetar in view of its high proper motion directed away from the Magnetar region, viz. the Luminous Blue Variable W243. We discuss the properties of W243 and how they pertain to the former Magnetar companion hypothesis. Binary evolution arguments are employed to derive a progenitor mass for the Magnetar of 24-25 $M_{\text {Sun }}$, just within the progenitor mass range for Neutron Star birth. We also draw attention to another candidate to be member of a former massive binary.

8th INTEGRAL Workshop "The Restless Gamma-ray Universe"

Dublin, Ireland

September 27-30, 2010

\footnotetext{
$1 \quad$ Speaker
} 


\section{Westerlund 1}

Westerlund $1(\mathrm{Wd} 1)$ is a Galactic star cluster of almost Super Star Cluster dimensions. On the basis of a comprehensive photometric study, Brandner et al. (2008) estimate for Wd1 a mass of $\sim 4.510^{4} M_{\text {Sun }}$, an age of 3.6 Myr and a distance of $3.6 \mathrm{kpc}$. Piatti et al. (1998) found a large obscuration of 12.9 magn $\left(A_{V}\right)$.

The cluster harbours a Magnetar, CXOU J164710.2-455216 (Figure 1), without an optical or Near-IR counterpart (Muno et al. 2006). This is the only collapsed object known in Wd1. Depending on the maximum stellar mass still yielding a Neutron Star remnant after Supernova explosion, an extrapolation of the cluster's likely Initial Mass Function indicates that some $10^{2}$ Supernovae leaving Black Hole remnants could have preceded the Magnetar (Brandner et al. 2008; Clark et al. 2008).

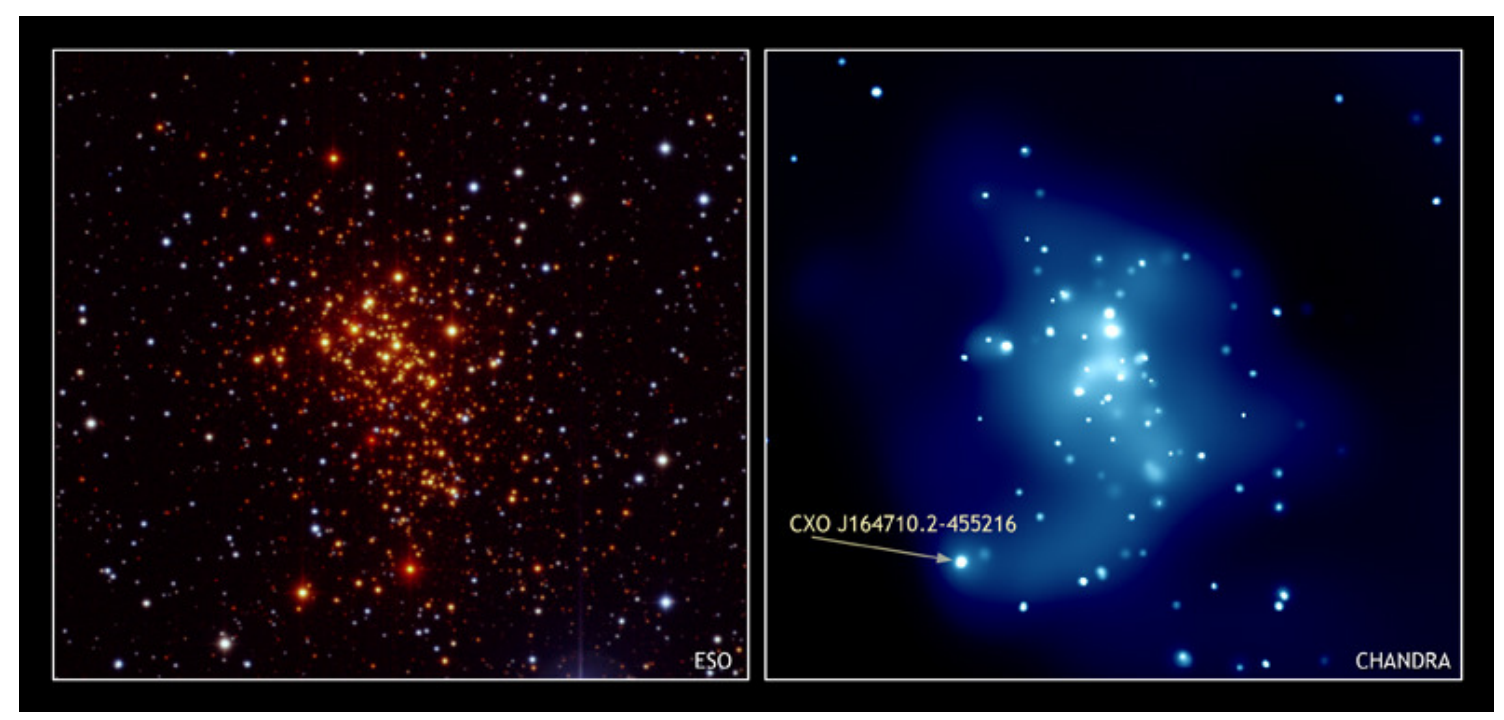

Figure 1. VLT optical image (left) and Chandra X-ray image (right) of Westerlund 1, the latter with Magnetar position indicated. (NASA/CXC/UCLA/Muno et al. 2006).

\section{Candidate former companion star W243}

The A-type supergiant W243 in Wd1 is found not far from the Magnetar. This star is a Luminous Blue Variable (LBV), with spectrum A2Iab. It displays spectral peculiarities and possesses a high proper motion $(222 \mathrm{~km} / \mathrm{s}$; UCAC2, Zacharias et al. 2003), directed away from the Magnetar (Figure 2).

As most massive stars are born in binaries, with mass ratios (q) towards unity, W243 presents itself as a candidate former companion to the Magnetar, to have been part of a binary disrupted after the Supernova explosion of the Magnetar progenitor. Indeed, a disrupted binary has been mentioned previously as a possibility for the Magnetar (Clark et al. 2008; Belczynski \& Taam 2008). 


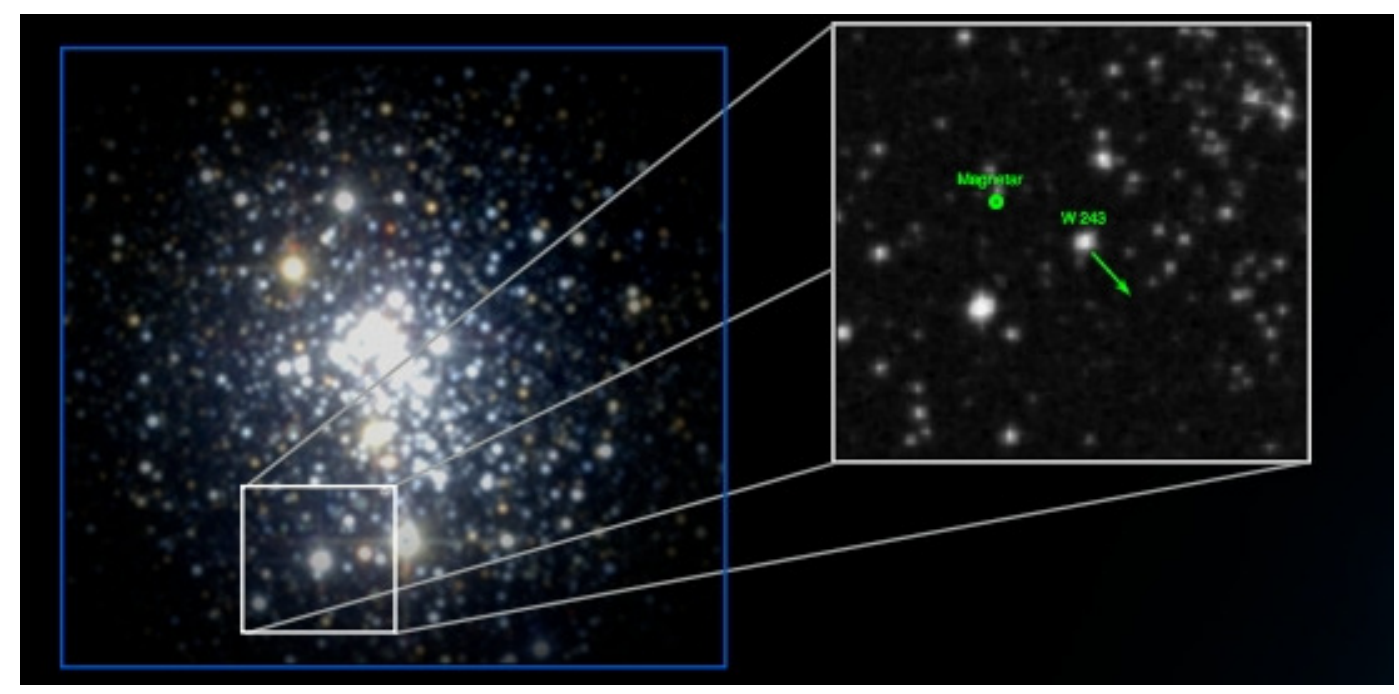

Figure 2. 2MASS image of Westerlund 1 core region (left) and Palomar Observatory Sky Survey IR image of W243 and surrounding region (right). The arrow in the right panel represents the proper motion of W243.

\section{On the binarity of $\mathrm{W} 243$}

In view of some of the spectral features of W243, an unseen hot companion (OB star) has been suggested for W243 (Ritchie et al. 2009). However, no period was found from optical monitoring of the star (Bonanos 2007).

We carried out spectro-astrometry employing 2 epochs of VLT-UVES spectra and find no evidence of a companion beyond a 25 AU binary separation (Figure 3 ). If indeed a hot companion is within this separation, the colliding stellar winds of the massive stars would be Xray bright. Assuming the model of Portegies Zwart et al. (2002) for an LBV system with low velocity winds and mass loss, such a binary system exhibits an X-ray luminosity of $>10^{34} \mathrm{erg} / \mathrm{s}$. No such luminous source is seen in Chandra or XMM-Newton observations of the cluster (Clark et al. 2008). Thus, we assume W243 to be a single runaway star.

\section{Binary history of Magnetar}

As in O'Maoileidigh et al. (2005), assuming (i) mass transfer stripping the $\mathrm{H}$ atmosphere of the Magnetar progenitor, (ii) a mass transfer efficiency $\propto \mathrm{q}^{2}$ (which is customary), and (iii) an initial mass ratio $\mathrm{q} \sim 0.89$ (for an evolved secondary, as is W243), we can estimate a progenitor mass for the Magnetar. For a post mass transfer W243 mass of $\sim 35-36 M_{\text {Sun }}$ (based on comparison with evolutionary tracks for rotating stellar models in Meynet \& Maeder 2000), we find for the Magnetar progenitor a mass of $\sim 24-25 M_{\text {Sun }}$.

This is lower than a recent suggestion of $M_{\text {initial }}>40 M_{\text {Sun }}$ for the progenitor (Ritchie et al. 2010). The difference with this value is largely due to these authors having used too large a distance to $\mathrm{Wd} 1, \sim 5 \mathrm{kpc}$, which has been more accurately determined by Brandner et al. (2008) as $3.6 \mathrm{kpc}$. Thus, luminosities and stellar masses had been overestimated due to the large distances. 


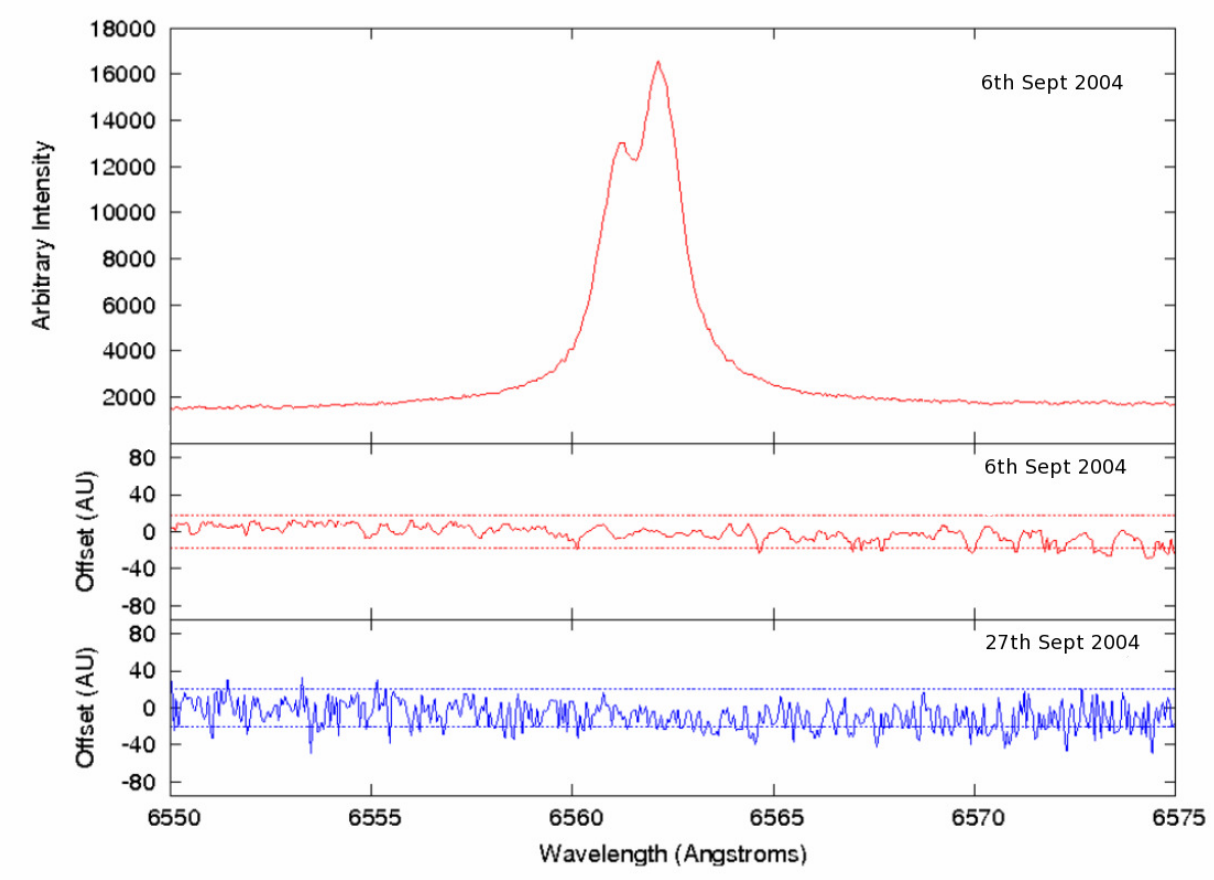

Figure 3. Spectro-photometric plot of the H $\alpha$ line in the W243 VLT-UVES spectra. The top panel shows the $\mathrm{H} \alpha$ region of the spectrum obtained on 6 Sept. 2004. The bottom two panels show the variation in the continuum centroid (in AU) across the wavelength range for each of the two observations, with the dotted lines representing the $1 \sigma$ error. If a binary companion were present one would expect a significant deviation in the centroid, offset across the $\mathrm{H} \alpha$ line.

Interestingly, the possibility of a 24-25 $M_{\text {Sun }}$ progenitor for the Magnetar is right at the upper limit determined by Heger et al. (2003), $25 M_{\text {Sun }}$, for Neutron Star formation from single stars, for metallicities not much exceeding the Solar value. For binaries the upper initial mass for a star leaving a Neutron Star after exploding as a Supernova could be a little lower, like $22 M_{\text {Sun }}$ (Fryer et al. 2002). Thus, the Magnetar could indeed be the first Neutron Star to have formed in Wd1 (after Black Holes) as lower and lower mass stars undergo a Supernova event as the cluster evolves with time, without challenging current estimated mass constraints on Neutron Star birth (cf. Richie et al. 2010). Confirmation of this picture may be obtained by a future proper motion measurement for the Magnetar. We note that the Main Sequence turn-off point of Wd1 may be around spectral types O7V-O8V (Muno et al. 2006), where O8V stars have masses of $\sim 23 M_{\text {Sun }}$ (Schmidt-Kaler 1982). However, from the analysis of Brandner et al. (2008) would follow still a lower turn-off mass, around $15 M_{\text {Sun }}$ (B0.5V stars).

\section{Another W243-like case in Wd1?}

Just NE of the centre of Wd1 is another runaway star, W16a, closely resembling W243. Its spectrum is A2Ia+ (or possibly A5Ia+) and the B, $\mathrm{V}$ and $\mathrm{J}$ magnitudes of these two stars are very similar. The proper motion of W16a is $188 \mathrm{~km} / \mathrm{s}$ (UCAC2, Zacharias et al. 2003). Thus, W16a could similarly have been part of a binary that was disrupted after its faster evolving companion underwent a Supernova event, presumably leaving a Black Hole. On the other hand, 
this star could also be an alternative candidate for a former companion to the Magnetar. Again, the proper motion of the Magnetar will be helpful to decide here.

\section{References}

[1] A.Z. Bonanos, Variability of Young Massive Stars in the Galactic Super Star Cluster Westerlund 1, AJ 133, 2696 (2007)

[2] W. Brandner, J.S. Clark, A. Stolte, R. Waters, I. Negueruela, S.P. Goodwin, Intermediate to low-mass stellar content of Westerlund 1, A\&A 478, 137 (2008)

[3] Clark, J. S.; Muno, M. P.; Negueruela, I.; Dougherty, S. M.; Crowther, P. A.;

Goodwin, S. P.; de Grijs, R., Unveiling the X-ray point source population of the Young Massive Cluster Westerlund 1, A\&A 477, 147 (2008)

[4] C.L. Fryer, A. Heger, N. Langer, S. Wellstein, The limiting stellar initial mass for Black Hole formation in close binary systems, ApJ 578, 335 (2002)

[5] A. Heger, C.L. Fryer, S.E. Woosley, N. Langer, D.H. Hartmann, How Massive single stars end their life, ApJ 591, 288 (2003)

[6] C. O'Maoileidigh, E.J.A. Meurs, L. Norci, On the feasibility of detection of neutron star companions to OB runaways using Gaia astrometry, NewA 10, 591 (2005)

[7] G. Meynet, A. Maeder, Stellar evolution with rotation. V. Changes in all the outputs of massive star models, A\&A 361, 101 (2000)

[8] M.P. Muno, J.S. Clark, P.A. Crowther, S.M. Dougherty, R. de Grijs, C. Law, S.L.W. McMillan, M.R. Morris, I. Negueruela, D. Pooley, S. Portegies Zwart, F. Yusef-Farhad, A Neutron Star with a Massive Progenitor in Westerlund 1, ApJ 636, L41 (2006)

[9] A.E. Piatti, E. Bica, J.J. Claria, Fundamental parameters of the highly reddened young open clusters Westerlund 1 and 2, A\&AS 127, 423 (1998)

[10] B.W. Ritchie, J.S. Clark, J. S., I. Negueruela, F. Najarro, Spectroscopic monitoring of the luminous blue variable Westerlundl-243 from 2002 to 2009, A\&A 507, 1597 (2009)

[11] B.W. Ritchie, J.S. Clark, I. Negueruela, N. Langer, A VLT/FLAMES survey for massive binaries in Westerlund 1. II. Dynamical constraints on magnetar progenitor masses from the eclipsing binary W13, A\&A 520, A48 (2010)

[12] T. Schmidt-Kaler, Basic Stellar Data, in Landolt-Börnstein: Numerical Data and Functional Relationships in Science and Technology - New Series, Bd VI/2b, p. 30 (1982)

[13] N. Zacharias, S.E. Urban, M.I. Zacharias, G.L. Wycoff, D.M. Hall, M.E. Germain, E.R. Holdenried, L. Winter, The Second U.S. Naval Observatory CCD Astrograph Catalog (UCAC2), yCat 1289,0 (2003) 